HIP-2001-05/TH

\title{
Proton stability in TeV-scale GUTs
}

\author{
Archil B. Kobakhidze ${ }^{\mathrm{a}, \mathrm{b}}$

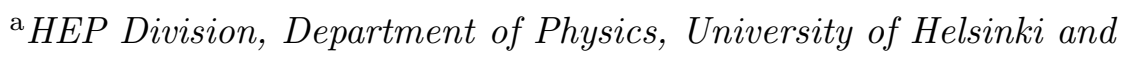 \\ Helsinki Institute of Physics, FIN-00014 Helsinki, Finland \\ ${ }^{\mathrm{b}}$ Andronikashvili Institute of Physics, GE-380077 Tbilisi, Georgia
}

\begin{abstract}
We discuss the proton decay problem in theories with low gravity and/or GUT scales. We pointed out that the gravity induced proton decay can be indeed suppressed up to a desired level, while the GUT origin of the proton instability is rather problematic. To solve this problem we suggest the GUT model where the proton is stable in all orders of perturbation theory. This can be simply achieved by the replication of quark-lepton families with ordinary quarks and leptons residing in different GUT representations and by an appropriate dimensional reduction. The model predicts extra mirror states which along with the GUT particles and the excitations of extra dimensions could be observable at high-energy colliders providing the unification scale is in the $\mathrm{TeV}$ range.
\end{abstract}


Over the few past years a great interest attract the Brane World models where the higherenergy scales such as a Planck scale [1, 2] and/or GUT scale [3, 4] are lowered down to the energies accessible for colliders'? Within these models new approaches to the long-standing problems of 4-dimensional particle physics such as hierarchy problem 11, 2, 6, 7], fermion masses [3, 8, 9, 10] etc. have been proposed. However, as it usually happens, these new scenarios brought new problems as well. Among them one is the potentially large and thus phenomenologically unacceptable violation of certain global symmetries in the low-energy effective theory [11]. This might be problematic for the realistic models since the strong violation of global symmetries could induce, say, large flavour changing neutral currents, large neutrino masses, unacceptably fast proton decay etc. In this note we will concentrate on the proton stability problem in GUTs with low scale unification [3, 4, 12].

Proton decay will take place if baryon $(B)$ and lepton $(L)$ numbers are simultaneously violated. The typical, lowest order (dimension 6) $B$ and $L$ violating operator can be written as:

$$
\frac{c}{\Lambda^{2}}(q q q l)
$$

where $q$ 's ere a quark fields and $l$ denote the leptons (an appropriate contraction of the internal and Lorentz indices in (1) is understood). The mass scale $\Lambda$ in the denominator of (1) is a typical energy scale where the $B$ and $L$ violation happens and the constant $c$ accounts a particular dynamics which leads to (1) at low-energies.

When two of $q$ 's in (1) denote up-quarks, while the third $q$ is a down one, and $l$ is an electron, the operator (11) can be responsible for the proton decay into the pion and positron: $p \rightarrow \pi^{0} e^{+}$. In many GUT models, for example, this is a dominant channel for the proton decay and the current experimental limit'

$$
\tau_{p \rightarrow \pi^{0} e^{+}} \gtrsim 1.6 \cdot 10^{33} y r
$$

puts strong constraints on the possible GUT extensions. Many simple non-supersymmetric GUTs are excluded (see, however, [13]) and the simplest $S U(5)$-type supersymmetric GUTs also fail to satisfy the bound (2) [14], unless some extra mechanism ensuring desired sup-

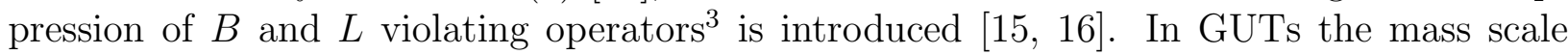
$\Lambda$ in (11) is associated with the scale of GUT-symmetry breaking, more precisely, with the masses of GUT particles mediating $B$ and $L$ violating interactions ( $X, Y$ gauge bosons and colored Higgs, and their superpartners in the case of supersymmetric GUT). These masses are expected to be of the order of unification scale, which for the supersymmetric GUTs turns out to be around $10^{16} \mathrm{GeV}$. Clearly, all conventional GUTs with $\Lambda<10^{15 \div 16} \mathrm{GeV}$ will inevitably face with the proton stability problem. Say, if the unification scale is around $\mathrm{TeV}$ [3, \&, 12], the proton life-time becomes $48 \div 52$ orders of magnitude smaller than the experimental upper bound quoted above (2).

\footnotetext{
${ }^{1}$ For an earlier proposal of TeV-scale extra dimensions see [5]. The higher-dimensional scales are effectively lowered in the Randall-Sundrum Brane World as well for the observer living on the so-called TeV-brane 6.

${ }^{2}$ The partial life-times of proton for other channels are also in the range given in (2).

${ }^{3}$ In supersymmetric GUTs the dominant contribution to the proton decay comes from the dimension 5 operators induced by exchange of the colored Higgsino.
} 
Moreover, there could be also another, non-GUT origin of the effective operators (四) responsible for the proton decay which we would like to discuss now. It is widely discussed that the gravity itself can be responsible for the violation of global numbers [17, 18, 19, 20, 21, 22, 23 including those of $B$ and $L$ [18, 19. One can distinguish two potential gravitational sources of proton decay: one is mediated by a virtual black hole and another is that due the wormhole background. For the conventional Planck scale $M_{P l} \simeq 10^{19} \mathrm{GeV}$ the life-time of proton decaying gravitationally is about 12 orders of magnitude less then upper experimental limit (2) and thus proton can be treated as practically stable. But now, if the fundamental Plank scale is actually $\mathrm{TeV}$, rather then $10^{19} \mathrm{GeV}$, as it is proposed in [1], 2, 3], the disaster at first glance will be inevitable [24]. We would like to stress here, however, that the conclusions of Ref. [24] is by far less obvious. Contrary to [24], with our present knowledge of black hole and wormhole physics, one can equally well motivate, that the violation of global quantum numbers by gravity is extremely suppressed, even if the fundamental Planck scale is as low as a few TeV.

The typical argumentation for the proton decay by a virtual black holes is as follows. The proton, viewing as a hollow sphere of a radius $r \sim m_{\text {proton }}^{-1} \approx 10^{-13} \mathrm{~cm}$ with three valence quarks inside, can transmute into another species of particles. This might happen when, say, two of the valence quarks fall into the black hole which subsequently evaporates due to the Hawking radiation [25]. Since the Hawking radiation is formed outside the black hole horizon, it is totally insensitive to the global numbers carried by quarks inside the horizon, and recognizes their local charges only, such as electric charge and colour. So, among the other light particles, black hole can emit the corresponding quark-lepton pair as well and thus the global baryon charge get lost, while the lepton charge appears from "nothing". This process can be formally (but not equivalently) viewed as the process induced by the exchange of a particle with mass $M_{P l}$, and can be described by the effective operator (11), where $\Lambda=M_{P l}$. However, the picture described above is rather questionable since the formation and subsequent evaporation of a black hole contradicts to the basics of quantum mechanics such as quantum mechanical determinism. This puzzle, also known as a black hole information loss paradox, is a subject of intensive debates during many years, but it still far from a complete resolution [20]. It is rather reasonable to think that the solution of this puzzle within a consistent theory of quantum gravity could automatically lead to the conservation of the global charges or to the substantial suppression of the nucleon decay rate (see e.g., [19]). So we can at least say, that so far there is no firm theoretical ground to indisputably believe that the global symmetries are indeed violated by a virtual black holes. Thus, the constraints such as discussed in [24] can not be viewed as a critical theoretical argument against the models with low scale gravity [1, 2].

The situation with wormholes is more clear. It was argued that the wormholes also lead to the violation of global charge conservation [21, 22, 23], but unlike the black holes this does not cause the information loss paradox. The difference is that in the case of wormholes there appear an effective coupling constant for the transition rate from one initial pure state to a different final pure state. These transitions look very similar to those which take place in the instanton background. So, the operators (11) responsible for the proton decay seems 
to indeed appear in the wormhole background and the constant $c$ in (1) is proportional to the (non-perturbative) exponential factor

$$
c \sim e^{-S}
$$

where $S$ is a wormhole action. Now, if $S \gtrsim 59$ we are perfectly safe to satisfy the bound (2) even if the fundamental Planck scale is around TeV, $\Lambda \sim M_{P l} \sim \mathrm{TeV}$. So, the question is: can we naturally obtain such a large values for the wormhole action $S$ ? The calculations performed in 23] show that most likely this is not the case within the pure 4-dimensional Einstein-Hilbert gravity $(S \sim \mathcal{O}(10))$. However, the wormhole action turns out to be very sensitive to the modifications of the Einstein-Hilbert gravity. Particularly, in the case of extra dimensions with relatively large compactification radii $R_{c} M_{P l} \sim \mathcal{O}(10)$ the action can be as large as $S \sim \mathcal{O}(100)$ d23. Thus the large extra dimensions lead to the substantial increase of the wormhole action and, as a consequence, to the dramatic suppression of the global charge violating processes. But this is precisely the picture we have within the $\mathrm{TeV}$ scale gravity models, where at least two of extra dimensions should have even hierarchically larger radii $\left(R_{c} \sim \mathrm{mm}\right)$ compared to the fundamental $\mathrm{TeV}$-scale in order to account the apparent weakness of gravity in the visible 4-dimensional world [1, 2]. Thus one can expect that even in the case when the fundamental Planck scale is as low as TeV the wormhole induced non-conservation of global numbers are safely suppressed due to the large wormhole action. From the above discussion we conclude that gravity induced proton decay can be indeed suppressed up to a desired level even for the fundamental Planck scale in the TeV range, while the GUT origin of the proton instability is rather problematic.

Meanwhile, some mechanisms to suppress potentially large violation of global charges in theories with low Planck/GUT scale have been recently suggested [2, 3, 9, 11, 26]. Basically they can be divided into two classes. One is the "conventional" approach relies on the gauging of corresponding global symmetries say that of baryon number [2], flavour symmetry [1], or on the extra gauge symmetries invoked to ensure proton stability [26]. However, most of the proposed gauge symmetries can not be straightforwardly extended to the GUT framework, since they treat quarks and leptons differently. Another approach is intrinsically higher dimensional and relies either on imposition of non-trivial boundary conditions on GUT fields within the framework of orbifold compactification [3] lepton fields on a thick 3-brane at a different points along the extra dimension [9]. These scenarios, being compatible with GUTs, require the chiral matter (i.e. ordinary quarks and leptons) to be stuck at a 4-dimensional hypersurface (3-brane) if the unification is assumed to be around the $\mathrm{TeV}$ scale. Indeed, in the scenario of Ref. [3], for example, if one allows the third family of quarks and leptons to freely propagate in extra dimensions, then even if the light families are localized on a 3-brane, the proton decay mediated by $X, Y$ bosons and colored Higgs (Higgsino) takes place due to the mixing of light quarks with those of top and

\footnotetext{
${ }^{4}$ The main contribution to the wormhole action is proportional to $\sim r^{2} M_{P l}^{2}$, where $r$ is the size of the wormhole throat. In the presence of extra dimension of radius $R_{c}, r$ could be of the order of $R_{c}$.

${ }^{5}$ Further recent discussions on non-trivial boundary conditions within the orbifold compactification in connection with GUT model building and supersymmetry breaking can be found in [27] and 28], respectively.
} 
bottom. The proton stability in this case demands the unification scale greater than $10^{12}$ $\mathrm{GeV}$ [3].

Here we propose an alternative possibility to ensure proton stability in low scale GUTs. Namely, we will discuss below an explicit and rather simple model based on $S U(5)$ gauge symmetry where the proton is stable in all orders of perturbation theoryl. Note once again that from the above discussion follows that it is sufficient to keep global $B$ or $L$ symmetries exact in order to have a long-lived proton.

Let us consider supersymmetric $S U(5)$ model in higher-dimensional space-time. For simplicity we restrict our discussion by the case of one compact extra space-like dimension with a certain topology. Since we are considering a supersymmetric theory in five dimensions, we have the model invariant under the $N=2$ supersymmetry in the flat uncompactified limit, and thus it is vector-like. Now, depending on the topology of extra dimension and transformation properties of the superfields involved, the theory can be arranged in such a way that after dimensional reduction one gets either $N=2$, or $N=1$ supersymmetric theory or even a non-supersymmetric theory. In the later cases one can also project mirror states thus obtaining a chiral theory in four dimensions.

As usually, each family of ordinary quarks and leptons is placed in $\overline{5}$ and 10 irreducible representations of the $S U(5)$ group but now the ordinary quarks and leptons are supplemented by the mirror states. The mirror states combine with ordinary quarks and leptons to form $N=2$ hypermultiplets:

$$
\begin{gathered}
\mathcal{Q}=\left(Q_{L}, Q_{R}\right) \sim \overline{5}, \\
\mathcal{D}=\left(D_{L}, D_{R}\right) \sim 10,
\end{gathered}
$$

Here $Q_{L}\left(D_{L}\right)$ and $Q_{R}\left(D_{R}\right)$ are $N=1$ left-handed and right-handed chiral quintuplet (decuplet) superfields, respectively, the scalar components (as well as auxiliary fields) of which form doublets of a global $S U(2)_{R} R$-symmetry of $N=2$ supersymmetry. The decomposition of the fields in (4, 5) under the $S U(3)_{C} \otimes S U(2)_{W} \otimes U(1)_{Y}$ Standard Model gauge group is familiar:

$$
\begin{aligned}
Q_{L(R)} & =\bar{d}_{L(R)} \sim\left(\overline{3}, 1,-\sqrt{\frac{2}{15}}\right)+l_{L(R)} \sim\left(1, \overline{2}, \sqrt{\frac{3}{20}}\right) \\
D_{L(R)} & =\bar{u}_{L(R)} \sim\left(\overline{3}, 1, \frac{2}{\sqrt{15}}\right)+q_{L(R)} \sim\left(3,2,-\frac{1}{\sqrt{60}}\right)+ \\
\bar{e}_{L(R)} & \sim\left(1,1,-\frac{3}{\sqrt{15}}\right) .
\end{aligned}
$$

\footnotetext{
${ }^{6}$ In fact, even the "minimal model" discussed in [3] is not fully satisfactory, because while the $X$ and $Y$ gauge bosons are decoupled from the ordinary quarks and leptons owing to the orbifold projection, their $N=2$ scalar partners do propagate on a 3-brane. Being coupled with quarks and leptons there, they also can mediate unacceptably rapid proton decay.

${ }^{7}$ For earlier GUT models with absolutely stable proton see e.g. 29].
} 
Note that so far there is no symmetry at hand which suppresses the direct mass terms for the quintuplets (困) $M_{\mathcal{Q}}$ and decuplets (5) $M_{\mathcal{D}}$ and a priory these masses can be as large as the fundamental Planck scale $M_{P l}$.

$N=2 S U(5)$ vector supermultiplet

$$
\mathcal{V}=(V, \Phi)
$$

contains $N=14$-dimensional gauge superfield $V=\left(A^{\mu}, \lambda^{1}, X^{3}\right)$ as well as $N=1$ chiral superfield $\Phi=\left(\Sigma+i A^{5}, \lambda^{2}, X^{1}+i X^{2}\right)$ both in the adjoint representation of the $S U(5)$ group $\left(V=V^{a} T^{a}, \Phi=\Phi^{a} T^{a}\right.$, where $a=1, \ldots, 24$ and $T^{a}$ are the $S U(5)$ generators in fundamental representation, $\left.\operatorname{Tr} T^{a} T^{b}=\frac{1}{2} \delta^{a b}\right)$ with the following decomposition:

$$
\begin{aligned}
V & =G \sim(8,1,0)+W \sim(1,3,0)+X \sim\left(\overline{3}, 2,-\sqrt{\frac{5}{12}}\right)+ \\
\bar{X} & \sim\left(3,2, \sqrt{\frac{5}{12}}\right)+S \sim(1,1,0) \\
\Phi & =\Phi_{G} \sim(8,1,0)+\Phi_{W} \sim(1,3,0)+\Phi_{X} \sim\left(\overline{3}, 2,-\sqrt{\frac{5}{12}}\right)+ \\
\Phi_{\bar{X}} & \sim\left(3,2, \sqrt{\frac{5}{12}}\right)+\Phi_{S} \sim(1,1,0)
\end{aligned}
$$

The gaugino $\lambda^{1}$ and the Higgsino $\lambda^{2}$ form an $S U(2)_{R}$ doublet, while auxiliary fields $X^{1,2,3}$ transform as $S U(2)_{R}$ triplet. Finally, we also need to introduce at least one $S U(5)$ fundamental and one anti-fundamental hypermultiplets:

$$
\begin{gathered}
\mathcal{H}=\left(H_{L}, H_{R}\right) \sim 5, \\
H_{L(R)}=h_{L(R)}^{C} \sim\left(3,1, \sqrt{\frac{2}{15}}\right)+h_{L(R)}^{W} \sim\left(1,2,-\sqrt{\frac{3}{20}}\right), \\
\widetilde{\mathcal{H}}=\left(\widetilde{H}_{L}, \widetilde{H}_{R}\right) \sim \overline{5}, \\
\widetilde{H}_{L(R)}=\widetilde{h}_{L(R)}^{C} \sim\left(\overline{3}, 1,-\sqrt{\frac{2}{15}}\right)+\widetilde{h}_{L(R)}^{W} \sim\left(1,2, \sqrt{\frac{3}{20}}\right),
\end{gathered}
$$

respectively, where the electroweak Higgs doublet (anti-doublet) $h_{L(R)}^{W}\left(\widetilde{h}_{L(R)}^{W}\right)$ presumably resides. $\mathcal{H}$ and $\widetilde{\mathcal{H}}$ could also have $S U(5)$-invariant masses $M_{\mathcal{H}}$ and $M_{\tilde{\mathcal{H}}}$, respectively. One obvious advantage of the $N=2$ supersymmetric GUTs is that the gauge fields in $V$ and scalars in $\Phi$ are unified in the same $N=2$ vector supermultiplet (8). The scalar component 
of the chiral superfield $\Phi$ can be used to break $S U(5)$ gauge symmetry down to the $S U(3)_{C} \otimes$ $S U(2)_{W} \otimes U(1)_{Y}$ so we do not need to introduce extra adjoint hypermultiplet. Certainly, in this respect, the $N=2$ models are much more predictive than the ordinary $N=1$ $S U(5)$. Alternatively, one can break $S U(5)$ invariance through the orbifold compactification [3], 27]. Having determined the particle content of the model, an $S U(5)$ and $N=2$ invariant 5-dimensional Lagrangian can be written down as [30]:

$$
\begin{gathered}
\mathcal{L}=\mathcal{L}^{\text {gauge }}+\mathcal{L}^{\text {matter }}, \\
\mathcal{L}^{\text {gauge }}=\frac{1}{4 g_{G U T}^{2}} \int d^{2} \theta \mathcal{W}^{2}+\text { h.c. }+\frac{1}{g_{G U T}^{2}} \int d^{4} \theta\left(\partial_{5} V-\frac{1}{\sqrt{2}}\left(\Phi+\Phi^{+}\right)\right)^{2}, \\
\mathcal{L}^{\text {matter }}=\int d^{4} \theta\left(\Psi_{L}^{+} \widehat{K} \Psi_{L}+\Psi_{R} \widehat{K}^{-1} \Psi_{R}^{+}\right)+\int d^{2} \theta \Psi_{R}^{+} \widehat{M} \Psi_{L}+\text { h.c },
\end{gathered}
$$

where $\mathcal{W}$ is an ordinary strength superfield of the $S U(5)$ gauge field, $g_{G U T}$ is an unified gauge coupling constant and

$$
\begin{gathered}
\Psi_{L(R)}=\left(Q_{L(R)}, D_{L(R)}, H_{L(R)}, \widetilde{H}_{L(R)}\right)^{T}, \\
\widehat{K}=\operatorname{diag}\left(e^{-V_{\mathcal{Q}}}, e^{-V_{\mathcal{D}}}, e^{-V_{\mathcal{H}}}, e^{-V_{\tilde{\mathcal{H}}}}\right), \\
\widehat{M}=\operatorname{diag}\left(\partial_{5}+M_{\mathcal{Q}}-\frac{1}{\sqrt{2}} \Phi_{\mathcal{Q}}, \partial_{5}+M_{\mathcal{D}}-\frac{1}{\sqrt{2}} \Phi_{\mathcal{D}}, \partial_{5}+M_{\mathcal{H}}-\frac{1}{\sqrt{2}} \Phi_{\mathcal{H}}, \partial_{5}+M_{\tilde{\mathcal{H}}}-\frac{1}{\sqrt{2}} \Phi_{\widetilde{\mathcal{H}}}\right) .
\end{gathered}
$$

Here $V_{\mathcal{Q}, \mathcal{D}, \mathcal{H}, \widetilde{\mathcal{H}}}\left(\Phi_{\mathcal{Q}, \mathcal{D}, \mathcal{H}, \widetilde{\mathcal{H}}}\right)=V^{a}\left(\Phi^{a}\right) T_{\mathcal{Q}, \mathcal{D}, \mathcal{H}, \widetilde{\mathcal{H}}}^{a}$, and $T_{\mathcal{Q}, \mathcal{D}, \mathcal{H}, \widetilde{\mathcal{H}}}^{a}$ are the $S U(5)$ generators where subscript refers to the corresponding representation. The usual repetition of quark-lepton generations residing in $Q_{L(R)}, D_{L(R)}$ is also assumed in the above equations.

The above Lagrangian (13,14,15) describes the minimal extension of the ordinary 4dimensional $N=1$ supersymmetric $S U(5)$ GUT to the case of five space-time dimensions. Clearly, the above model in many aspects is unacceptable from the phenomenological point of view. Then a certain dimensional reduction should provide the projection out of unwanted states at low-energies in lower dimensions. We will consider here the compactification of extra fifth dimension on an $S^{1} / Z_{2}$ orbifold. What we will require additionally is the conservation of $B$ and/or $L$ global charges upon the compactification. In fact this is not difficult to achieve. First, in addition to the particle content given above, let us introduce an extra quintuplet $\widetilde{\mathcal{Q}}$ $\sim \overline{5}$ and an extra decuplet $\widetilde{\mathcal{D}}, \sim 10$ of matter fields per each family of quarks and leptons. The second step is to appropriately project the different states in $\mathcal{Q}, \widetilde{\mathcal{Q}}, \mathcal{D}$, and $\widetilde{\mathcal{D}}$ upon the dimensional reduction. This can be done by assigning different $Z_{2}$ orbifold $Z_{2}$-numbers to the quarks and leptons (and their mirrors) in $\mathcal{Q}, \widetilde{\mathcal{Q}}, \mathcal{D}$, and $\widetilde{\mathcal{D}}$ as we will demonstrate just below.

${ }^{8}$ We consider here the model with minimal (quadratic) prepotential on the Coulomb branch. Such a minimal prepotential can be forced by the topology of compactified space 28]. 


\begin{tabular}{|c|c|c|}
\hline \multicolumn{2}{|l|}{ Fields } & $Z_{2}$ parity, $\mathcal{P}$ \\
\hline Vector supermultiplet, $\mathcal{V}$ & $\begin{array}{l}G\left(\Phi_{G}\right) \sim(8,1,0) \\
W\left(\Phi_{W}\right) \sim(1,3,0) \\
S\left(\Phi_{S}\right) \sim(1,1,0) \\
X,\left(\Phi_{X}\right) \sim\left(\overline{3}, 2,-\sqrt{\frac{5}{12}}\right) \\
\bar{X}\left(\Phi_{\bar{X}}\right) \sim\left(3,2, \sqrt{\frac{5}{12}}\right)\end{array}$ & $\begin{array}{l}+(-) \\
+(-) \\
+(-) \\
-(+) \\
-(+)\end{array}$ \\
\hline Quintuplets, $\mathcal{Q}, \widetilde{\mathcal{Q}}$ & $\begin{aligned} \bar{d}_{L(R)}, \widetilde{\bar{d}}_{L(R)} & \sim\left(\overline{3}, 1,-\sqrt{\frac{2}{15}}\right) \\
l_{L(R)}, \widetilde{l}_{L(R)} & \sim\left(1, \overline{2}, \sqrt{\frac{3}{20}}\right)\end{aligned}$ & $\begin{array}{l}-(+),+(-) \\
+(-),-(+)\end{array}$ \\
\hline Decuplets, $\mathcal{D}, \widetilde{\mathcal{D}}$ & $\begin{aligned} \bar{u}_{L(R)}, \widetilde{\bar{u}}_{L(R)} & \sim\left(\overline{3}, 1, \frac{2}{\sqrt{15}}\right) \\
q_{L(R)}, \widetilde{q}_{L(R)} & \sim\left(3,2,-\frac{1}{\sqrt{60}}\right) \\
\bar{e}_{L(R)}, \widetilde{\bar{e}}_{L(R)} & \sim\left(1,1,-\frac{3}{\sqrt{15}}\right)\end{aligned}$ & $\begin{array}{l}-(+),+(-) \\
+(-),-(+) \\
-(+),+(-)\end{array}$ \\
\hline Higgs hypermultiplets, $\mathcal{H}, \widetilde{\mathcal{H}}$ & $\begin{aligned} h_{L(R)}^{C} & \sim\left(3,1, \sqrt{\frac{2}{15}}\right) \\
h_{L(R)}^{W} & \sim\left(1,2,-\sqrt{\frac{3}{20}}\right) \\
\widetilde{h}_{L(R)}^{W} & \sim\left(1,2, \sqrt{\frac{3}{20}}\right) \\
\widetilde{h}_{L(R)}^{C} & \sim\left(\overline{3}, 1,-\sqrt{\frac{2}{15}}\right)\end{aligned}$ & $\begin{array}{l}-(+) \\
+(-) \\
+(-) \\
-(+)\end{array}$ \\
\hline
\end{tabular}

Table 1: An intrisic parity $\mathcal{P}$ of various fields (see eqs. (四11).

An intrinsic $Z_{2}$ parity $\mathcal{P}(= \pm 1)$ of a generic 5-dimensional bulk field $\varphi\left(x^{\mu}, y\right)$ is defined as:

$$
\varphi\left(x^{\mu},-y\right)=\mathcal{P} \varphi\left(x^{\mu}, y\right)
$$

Parity odd $\varphi_{+}(\mathcal{P}=1)$ and parity even $\varphi_{-}(\mathcal{P}=-1)$ can be expanded as:

$$
\begin{aligned}
& \varphi_{+}=\sum_{n=0}^{\infty} \varphi^{(n)}\left(x^{\mu}\right) \cos \left(n y / R_{c}\right) \\
& \varphi_{-}=\sum_{n=1}^{\infty} \varphi^{(n)}\left(x^{\mu}\right) \sin \left(n y / R_{c}\right),
\end{aligned}
$$

where $n$ is an integer number determining the quantized momentum corresponding to the extra compact dimension with radius $R_{c}$. A remarkable property of the compactification on $S^{1} / Z_{2}$ is that the parity-odd fields $\varphi_{-}$(21) lack zero modes. Now let us determine $Z_{2^{-}}$numbers of various fields involved in our model as it is given in Table 1 . Then it can be straightforwardly checked that the Lagrangian (13) is actually invariant under the orbifold parity transformations as it should bef. According to the expansion (20,21) the

\footnotetext{
${ }^{9}$ Note that the orbifold symmetry forbids the mass terms $M_{\mathcal{Q}}, M_{\mathcal{D}}$ and $M_{\mathcal{H}}$ in $(15)$. Also since $\Phi_{S}$ is
} 
wave functions of the parity-odd fields vanish at the orbifold fixed-points $(y=0, \pi)$ and only parity-even fields can propagate on the 4-dimensional boundary walls. This suggest the identification of ordinary quarks and leptons with $\mathcal{Q}, \widetilde{\mathcal{Q}}, \mathcal{D} \widetilde{\mathcal{D}}$ fragments as:

$$
\widetilde{\bar{d}}_{L}, \widetilde{\bar{u}}_{L}, q_{L}, l_{L}, \widetilde{\bar{e}}_{L}
$$

There also present their mirror states on the boundary wall:

$$
\bar{d}_{R}, \bar{u}_{R}, \widetilde{q}_{R}, \widetilde{l}_{R}, \bar{e}_{R}
$$

The gauge symmetry on the wall is just $S U(3)_{C} \otimes S U(2)_{W} \otimes U(1)_{Y}$ one and $N=2$ supersymmetry is reduced to the $N=1$. The vector superfields $X, \bar{X}$ from (9) as well as chiral superfields $\Phi_{G}, \Phi_{W}, \Phi_{S}$ from (10) are projected out since all they are $Z_{2}$-odd. In the same way coloured triplet (anti-triplet) superfield $h_{L}^{C}\left(\widetilde{h}_{L}^{C}\right)$ decouples from the electroweak doublet (anti-doublet) $h_{L}^{W}\left(\widetilde{h}_{L}^{W}\right)$ on the boundary wall, thus realizing intrinsically higher-dimensional mechanism for the doublet-triplet splitting. Beside the mirror states (23) we have some additional states beyond the usual particle content of the minimal supersymmetric Standard Model (MSSM). They are chiral leptoquark superfields $\Phi_{X}$ and $\Phi_{\bar{X}}$ from (10) and the colored triplet and anti-triplet anti-chiral superfields $h_{R}^{C}, \widetilde{h}_{R}^{C}$ from (11) and (12), respectively.

Now, since the gauge superfields $X, \bar{X}$ are $Z_{2}$-odd, they decoupled from the zero modes of quarks and leptons and thus they can not be responsible for the $B$ and $L$ violating interactions among them anymore. The adjoint scalar superfields $\Phi_{X}$ and $\Phi_{\bar{X}}$, contrary, have a zero modes and couple to the matter on the boundary wall. However, they can only transform the mirror quarks into the ordinary leptons and the mirror leptons into the ordinary quarks (see eqs. (15,18)). So among the possible final states along with the ordinary quarks and leptons always will appear the mirror ones. This actually means that in the limit of massless ordinary quarks and leptons and their mirror partners the following global charges are separately conserved:

$$
\begin{aligned}
& Q_{1}=B+L_{M}, \\
& Q_{2}=L+B_{M},
\end{aligned}
$$

where the mirror baryon and lepton numbers we denote as $B_{M}$ and $L_{M}$, respectively. To generate the masses for the ordinary quarks and leptons and their mirror partners we add $N=2$ supersymmetry violating terms to the Lagrangian (13) on the boundary wall at $y=0$ :

$$
\begin{aligned}
\mathcal{L}^{Y \text { ukawa }}= & \int d^{2} \theta \delta(y)\left[Y^{(\text {lept. })} Q_{L} \widetilde{D}_{L} \widetilde{H}_{L}+Y^{(\text {down })} \widetilde{Q}_{L} D_{L} \widetilde{H}_{L}+Y^{(\text {up })} D_{L} \widetilde{D}_{L} H_{L}+\right. \\
& \left.Y_{M}^{(\text {lept. })} \widetilde{Q}_{R} D_{R} H_{L}^{+}+Y_{M}^{(\text {down })} Q_{R} \widetilde{D}_{R} H_{L}^{+}+Y_{M}^{(\text {up })} D_{R} \widetilde{D}_{R} \widetilde{H}_{L}^{+}+\text {h.c. }\right],
\end{aligned}
$$

$Z_{2}$-odd it can not acquire non-zero vacuum expectation value. The GUT symmetry is actually broken by the non-trivial boundary conditions (19) determined by $Z_{2}$-numbers from Table 1. 
where $Y^{(\ldots)}$ and $Y_{M}^{(\ldots)}$ are the Yukawa constants for the ordinary quarks, leptons and their mirror partners, respectively, and a certain family structure in (26) is assumed. Because the appearance of $\delta(y)$-term, only the zero modes of corresponding superfields appear in (26). This actually means that the colored Higgs triplets from $H_{L}$ and $\widetilde{H}_{L}$ completely decoupled from the quarks and leptons as well as from their mirror partners. Consequently, the interactions in (26) do not violate neither $B$ and $B_{M}$ nor $L$ and $L_{M}$ and $Q_{1}$ and $Q_{2}$ remain conserved $\square$. It is evident now that, since the mirror particles is assumed to be heavier than the ordinary ones, the proton decay is forbidden kinematically. In other words, as long as mirror particles cannot be produced $B$ and $L$ are separately conserved. As a result the proton is absolutely stable. We would like to stress once again that the above consideration is valid in all orders in perturbation theory.

Concluding, probably one can think, that the idea of low-scale gravity and unification is too radical to be true. What is really exciting, however, is that this idea have indeed survived under the pressure of phenomenological constraints. Moreover, the proposed solutions to the existing problems often give even more exotic predictions which can be tested in the visible future. One such model has been discussed in this paper. Motivated by the solution of the proton decay problem we have constructed GUT model where baryon $B$ and lepton $L$ numbers are perturbatively conserved. The model predicts extra mirror states $\square$ which along with the GUT particles and the excitations of extra dimensions could be observable at high-energy colliders providing the unification scale is in the $\mathrm{TeV}$ range. Thus remains to hopefully wait for new collider experiments.

Acknowledgments. I would like to thank Zurab Berezhiani and Ilia Gogoladze for useful discussions. This work was supported by the Academy of Finland under the Project No. 163394.

Note added After this work was completed the paper 32 appeared in the hep-archive where the proton decay problem is discussed within the $S U(5)$ model in five dimensions compactified on $S^{1} /\left(Z_{2} \times Z_{2}^{\prime}\right)$ orbifold.

\section{References}

[1] N. Arkani-Hamed, S. Dimopoulos and G. Dvali, Phys. Lett. B429 (1998) 263; I. Antoniadis, N. Arkani-Hamed, S. Dimopoulos and G. Dvali, Phys. Lett. B436 (1998) 257.

\footnotetext{
${ }^{10}$ The mirror neutrinos have to be massive as well. For this purpose one can introduce an extra $S U(5)$-singlet hypermultiplet $\mathcal{S}=\left(S_{L}, S_{R}\right)$ with $\mathcal{P}\left(S_{R}\right)=-\mathcal{P}\left(S_{R}\right)=1$. Then the coupling $\int d^{2} \theta \delta(y)\left[Y_{M}^{(\text {neut })} S_{R} \widetilde{Q}_{R} H_{L}^{+}+\right.$h.c. $]$will be responsible for the generation of the mirror neutrino mass after the electroweak symmetry breaking. Note that the above term also preserves $L_{M}$ and thus does not alter our discussion

${ }^{11}$ Previous models as well as phenomenology of mirror fermions can be found in [31].
} 
[2] N. Arkani-Hamed, S. Dimopoulos and G. Dvali, Phys. Rev. D59 (1999) 086004.

[3] K. R. Dienes, E. Dudas and T. Gherghetta, Phys. Lett. B436 (1998) 55; Nucl. Phys. B537 (1999) 47.

[4] D. Ghilencea and G. G. Ross, Phys. Lett. B442 (1998) 165; Z. Kakushadze, Nucl. Phys. B548 (1999) 205.

[5] I. Antoniadis, Phys. Lett. B246 (1990) 377.

[6] L. Randall and R. Sundrum, Phys. Rev. Lett. 83 (1999) 3370; Phys. Rev. Lett. 83 (1999) 4690.

[7] M. Chaichian and A.B. Kobakhidze, Phys. Lett. B478 (2000) 299; A.B. Kobakhidze, hep-th/0012191.

[8] S.A. Abel and S.F. King, Phys. Rev. D59 (1999) 095010; A.B. Kobakhidze, hep-ph/9904203; K. Yoshioka, Mod. Phys. Lett. A15 (2000) 29; M. Bando, T. Kobayashi, T. Noguchi and K. Yoshioka, Phys. Lett. B480 (2000) 187.

[9] N. Arkani-Hamed and M. Schmaltz, Phys. Rev. D61 (2000) 033005.

[10] G. Dvali and M. Shifman, Phys. Lett. B475 (2000) 295; D.E. Kaplan and T.M. Tait, JHEP. 0006 (2000) 020; S.J. Huber and Q. Shafi, hep-ph/0010195; M.V. Libanov and S.V. Troitsky, hep-ph/0011095.

[11] Z. Berezhiani and G. Dvali, Phys. Lett. B450 (1999) 24; Z. Kakushadze, Nucl. Phys. B551 (1999) 549.

[12] Z. Berezhiani, I. Gogoladze and A.B. Kobakhidze, in preparation.

[13] J.L. Chkareuli, I.G. Gogoladze and A.B. Kobakhidze, Phys. Lett. B340 (1994) 63; Phys. Lett. B376 (1996) 111; A.B. Kobakhidze, Phys. Lett. B430 (1998) 290.

[14] See e.g., J. Hisano, hep-ph/0004266 and references therein.

[15] G. Dvali, Phys. Lett. B287 (1992) 101; Phys. Lett. B372 (1996) 113; Z. Berezhiani, Phys. Lett. B355 (1995) 178; I. Gogoladze and A. Kobakhidze, Phys. Atom. Nucl. 60 (1997) 126; Z. Berezhiani, Z. Tavartkiladze and M. Vysotsky, hep-ph/9809301.

[16] J. Hisano, H. Murayama and T. Yanagida, Phys. Lett. B291 (1992) 263; K.S. Babu and S.M. Barr, Phys. Rev. D48 (1993) 5354; J. Hisano, T. Moroi, K. Tobe and T. Yanagida, Phys. Lett. B342 (1995) 138.

[17] S.W. Hawking, Nucl. Phys. B144 (1978) 349; G.W. Gibbons and S.W. Hawking, Phys. Rev. D15 (1977) 2752. 
[18] Ya.B. Zeldovich, Phys. Lett. A59 (1976) 254; Sov. Phys. JETP 45 (1977) 9; S.W. Hawking, D.N. Page and C.N. Pope, Phys. Lett. B86 (1979) 175.

[19] D.N. Page, Phys. Lett. B95 (1980) 244.

[20] For reviews see e.g., D.N. Page, hep-th/9305040; T. Banks, Nucl. Phys. Proc. Suppl. 41 (1995) 21; A. Strominger, hep-th/9501071.

[21] S.W. Hawking, Phys. Lett. B195 (1987) 337; G.V. Lavrelashvili, V.A. Rubakov and P.G. Tinyakov, JETP Lett. 46 (1987) 167.

[22] S. Giddings and A. Strominger, Nucl. Phys. B306 (1988) 349; Nucl. Phys. B321 (1989) 481; L.F. Abbot and M. Wise, Nucl. Phys. B325 (1989) 687; S. Coleman and K. Lee, Nucl. Phys. B329 (1989) 389.

[23] R. Kallosh, A. Linde, D. Linde and L. Susskind, Phys. Rev. D52 (1995) 912.

[24] F.C. Adams, G.L. Kane, M. Mbonye and M.J. Perry, hep-ph/0009154.

[25] S.W. Hawking, Nature 248 (1974) 30; Commun. Math. Phys. 45 (1975) 199.

[26] L.E. Ibañez and F. Quevedo, JHEP. 9910 (1999) 001; A. Aranda and C.D. Carone, hep-ph/0012092.

[27] Y. Kawamura, Prog. Theor. Phys. 103 (2000) 613; hep-ph/0012125; hep-ph/0012352.

[28] M. Chaichian, A.B. Kobakhidze and M. Tsulaia, hep-th/0101210.

[29] H. Fritzsch and P. Minkowski, Phys. Lett. B56 (1975) 69; M. Gell-Mann, P. Ramond, R. Slansky, Rev. Mod. Phys. 50 (1978) 721; P. Langacker, G. Segre and H.A. Weldon, Phys. Lett. B73 (1978) 87; Phys. Rev. D18 (1978) 552; P. Fayet, Phys. Lett. B153 (1985) 397; R.N. Mohapatra, Phys. Rev. D54 (1996) 5728.

[30] N. Arkani-Hamed, T. Gregoire and J. Wacker, hep-th/0101233.

[31] C. Csaki and F. Csikor, Phys. Lett. B309 (1993) 103; F. Csikor and I. Montvay, Phys. Lett. B324 (1994) 412; I. Montvay, hep-ph/9708269; G. Triantaphyllou, Eur. Phys. J. C10 (1999) 703; J. Phys. G26 (2000) 103; Int. J. Mod. Phys. A15 (2000) 265; H.J. He, N. Polonsky, S. Su, hep-th/0102144. For earlier works see also the review J. Maalampi and M. Roos, Phys. Rept. 186 (1990) 53, and references therein.

[32] G. Altarelli and F. Feruglio, hep-ph/0102301. 